\title{
Parametric quantum spin pump
}

\author{
Wei Zheng, ${ }^{1}$ Junling Wu, ${ }^{2}$ Baigeng Wang, ${ }^{3,2}$ Jian Wang, ${ }^{1,2, *}$ Qingfeng Sun, ${ }^{4}$ and Hong Guo ${ }^{4}$ \\ ${ }^{1}$ Institute of Solid State Physics, Chinese Academy of Sciences, Hefei, Anhui, People's Republic of China \\ ${ }^{2}$ Department of Physics, The University of Hong Kong, Pokfulam Road, Hong Kong, China \\ ${ }^{3}$ National Laboratory of Solid State Microstructures and Department of Physics, Nanjing University, Nanjing, \\ People's Republic of China \\ ${ }^{4}$ Department of Physics, McGill University, Montreal, PQ, H3A, 2T8, Canada
}

(Received 18 March 2003; published 24 September 2003)

\begin{abstract}
We investigate a nonadiabatic parametric quantum pump consisting of a nonmagnetic scattering region connected by two ferromagnetic leads. The presence of ferromagnetic leads allows electrons with different spins to experience different potential landscapes. Using this effect we propose a quantum spin pump that drives spin-up electrons to flow in one direction and spin-down electrons to flow in opposite direction. As a result, the spin pump can deliver a spin current with a vanishing charge current.
\end{abstract}

DOI: 10.1103/PhysRevB.68.113306

A parametric quantum pump generates a dc electric current by a cyclic variation of system parameters while keeping the leads at a constant chemical potential. ${ }^{1}$ Considerable effort has been devoted to understanding the physics of parametric pumping. ${ }^{2-8}$ It was found that the pumped current is rather sensitive to various parameters of the system such as the potential of the pump, the frequency of the driving force, and the Fermi energy of the leads. As parameters vary, the pumped current can even change directions: this has been predicted for charge pumping in resonant tunneling diodes, ${ }^{4,9}$ nanotube quantum pumps, ${ }^{10}$ finite frequency pumping process ${ }^{11}$ and quantization of the pumped charge. ${ }^{12}$ To fully exploit this behavior, in this paper we investigate parametric pumping in the presence of ferromagnetic leads. When ferromagnetic leads are present, electrons with different spins experience different potentials. Under certain conditions the pumped current for electron with different spin may flow in opposite directions. As a result, the total pumped charge current can be zero while a pure spin current is delivered. Such a quantum spin pump may have potential applications in the fascinating field of spintronics. ${ }^{13}$

Recently, several different spin pumps have been proposed. ${ }^{14-17}$ In the unipole spin battery studied in Refs. 14 and 15 , a spin current is generated by either a rotating magnetic moment or a rotating external magnetic field. By shining a microwave on a double quantum dot in the presence of a nonuniform magnetic field, a bipolar spin battery is proposed. ${ }^{16}$ In another direction, an adiabatic quantum pump was proposed ${ }^{17}$ such that a spin polarized current is generated in a chaotic quantum dot in the presence of an in-plane magnetic field. Indeed, in such a system the spin current has been generated experimentally ${ }^{18}$ as the magnetic field is varied. In this work we go beyond the adiabatic regime and examine the frequency dependence of the pumped spin current. Our results show that a pure spin current without charge current can be achieved by varying the pumping frequency.

The system we investigate is a spin-valve which consists of a two-dimensional nonmagnetic scattering region connected by two ferromagnetic electrodes to the reservoir through two narrow constrictions controlled by gate voltages. Since the threshold of electron propagation in the con-
PACS number(s): 72.25.Mk, 85.35.-p, 74.40.+k striction may be lower than that in the lead, the constrictions act like a double barrier whose height can be tuned by two gate voltages. The cyclic variation of these two pumping gate voltages allows parametric electron pumping through the quantum scattering region. We note that using the gate voltage to control the pumping potential was realized experimentally by Switkes et al. ${ }^{1}$ To simplify the calculation, we use an one-dimensional double barrier potential to model the quantum scattering region. The magnetic moment $\mathbf{M}$ of the left electrode is pointing in the $z$ direction, the electric current is flowing in the $y$ direction, while the moment of the right electrode is at an angle $\theta$ to the $z$ axis in the $x-z$ plane. For simplicity of discussion, we assume that the value of the molecular field $M$ is the same for the two electrodes; thus a standard spin-valve effect is obtained ${ }^{19}$ by varying the angle $\theta$. Essentially, $M$ mimics the difference of density of states between spin-up and -down electrons ${ }^{19}$ in the electrodes. The pumped current for this system can be calculated at a finite frequency and up to second order in pumping amplitude using the perturbation theory based on nonequilibrium Green's functions. ${ }^{11}$ The pumped particle current due to spin component $\sigma$ through lead $\alpha$ is found to be $(\hbar=1),{ }^{20}$

$$
\begin{aligned}
J_{\alpha \sigma}= & i \int \frac{d E}{2 \pi} \operatorname{Tr}\left[\boldsymbol { \Gamma } _ { \alpha } \mathbf { G } ^ { r } \left[\left(f_{-}-f\right) V\left(\mathbf{G}^{r}\left(E_{-}\right)-\mathbf{G}^{a}\left(E_{-}\right)\right) V^{*}\right.\right. \\
& \left.\left.+V^{*}\left(f_{+}-f\right)\left(\mathbf{G}^{r}\left(E_{+}\right)-\mathbf{G}^{a}\left(E_{+}\right)\right) V\right] \mathbf{G}^{a}\right]_{\sigma \sigma},
\end{aligned}
$$

where $\sigma=\uparrow, \downarrow, V$ is the pumping potential profile, ${ }^{20} E_{ \pm}=E$ $\pm \omega$, and $G^{r}$ is a $2 N \times 2 N$ ( $N$ for the orbital degrees of freedom and 2 is for spin) matrix representing the equilibrium retarded Green's function

$$
\mathbf{G}^{r}(E)=\frac{1}{E-H_{0}-\mathbf{\Sigma}^{r}},
$$

with $H_{0}$ the Hamiltonian in the absence of pumping potential. The trace is over orbital degrees only in Eq. (1). Here $\boldsymbol{\Sigma}^{r} \equiv \Sigma_{\alpha} \boldsymbol{\Sigma}_{\alpha}^{r}$ is the self energy and $\boldsymbol{\Gamma}_{\alpha}=-2 \operatorname{Im}\left[\boldsymbol{\Sigma}_{\alpha}^{r}\right]$ is the linewidth function. The self-energies are given by ${ }^{21}$ 


$$
\boldsymbol{\Sigma}_{\alpha}^{r}(E)=\hat{R}_{\alpha}\left(\begin{array}{cc}
\Sigma_{\alpha \uparrow}^{r} & 0 \\
0 & \Sigma_{\alpha \downarrow}^{r}
\end{array}\right) \hat{R}_{\alpha}^{\dagger},
$$

with the rotational matrix $\hat{R}_{\alpha}$ for electrode $\alpha$ defined as

$$
\hat{R}=\left(\begin{array}{cc}
\cos \theta_{\alpha} / 2 & \sin \theta_{\alpha} / 2 \\
-\sin \theta_{\alpha} / 2 & \cos \theta_{\alpha} / 2
\end{array}\right) .
$$

Here angle $\theta_{\alpha}$ is defined as $\theta_{L}=0$ and $\theta_{R}=\theta$ and $\Sigma_{\alpha \sigma}^{r}$ is the usual self energy. ${ }^{22}$ In this paper, we will study two special cases: $\theta=0$ or $\pi$ for spin current. From Eq. (1), we observe that up to the second order in pumping amplitude, the particle can absorb or emit a photon during the pumping process. ${ }^{11}$ The contribution due to these two photon assisted processes have different sign and tend to cancel each other. As the result of this competition, the pumped particle current can reverse its direction upon varying system parameters.

The pumped charge current $I_{\alpha}$ is given by

$$
I_{\alpha}=q\left(J_{\alpha \uparrow}+J_{\alpha \downarrow}\right),
$$

and the pumped spin current $I_{s \alpha}$ is (we have set $\hbar=1$ )

$$
I_{s \alpha}=\left(J_{\alpha \uparrow}-J_{\alpha \downarrow}\right) / 2 .
$$

Now we examine the conservation law for pumped current. When $\theta=0, \pi$, the $2 \times 2$ Green's function $\mathbf{G}^{r}$ and self energy are diagonal. As a result, we have

$$
\begin{aligned}
\sum_{\alpha} J_{\alpha \sigma}= & i \int \frac{d E}{2 \pi} \operatorname{Tr}\left[\mathbf{G}^{a} \boldsymbol{\Gamma} \mathbf{G}^{r}\left(f_{-}-f\right)\right. \\
& \times V\left(\mathbf{G}^{r}\left(E_{-}\right)-\mathbf{G}^{a}\left(E_{-}\right)\right) V^{*}+\mathbf{G}^{a} \mathbf{\Gamma} \mathbf{G}^{r}\left(f_{+}-f\right) \\
& \left.\times V^{*}\left(\mathbf{G}^{r}\left(E_{+}\right)-\mathbf{G}^{a}\left(E_{+}\right)\right) V\right]_{\sigma \sigma}
\end{aligned}
$$

where we have moved $\mathbf{G}^{a}$ to the beginning of the trace. This can be done only if the Green's function and self energy are diagonal. Using the fact that $\mathbf{G}^{r}-\mathbf{G}^{a}=-i \mathbf{G}^{a} \mathbf{\Gamma} \mathbf{G}^{r}$ and changing variable from $E_{+}$to $E$ in the second term of Eq. (7), we have

$$
\begin{aligned}
\sum_{\alpha} J_{\alpha \sigma}= & -\int \frac{d E}{2 \pi} \operatorname{Tr}\left[( \mathbf { G } ^ { r } - \mathbf { G } ^ { a } ) ( f _ { - } - f ) V \left(\mathbf{G}^{r}\left(E_{-}\right)\right.\right. \\
& \left.-\mathbf{G}^{a}\left(E_{-}\right)\right) V^{*}+\left(\mathbf{G}^{r}\left(E_{-}\right)-\mathbf{G}^{a}\left(E_{-}\right)\right) \\
& \left.\times\left(f-f_{-}\right) V^{*}\left(\mathbf{G}^{r}-\mathbf{G}^{a}\right) V\right]_{\sigma \sigma},
\end{aligned}
$$

which is zero since the Green's function and self energy are diagonal. This means that both the pumped electric current and pumped spin current are conserved in this device.

Now we use Eqs. (1), (5), and (6) to calculate the pumped electric current and the spin current. The system we studied is a symmetric double $\delta$ barrier structure modeled by a potential $U(x)=V_{0} \delta(x+a)+V_{0} \delta(x-a)$. For this system the Green's function $G\left(x, x^{\prime}\right)$ can be calculated exactly. ${ }^{23}$ The pumping potential is chosen to be sinusoidal, $V(x, t)$ $=V_{p}\left[\cos (\omega t) \delta\left(x-x_{1}\right)+\cos (\omega t+\phi) \delta\left(x-x_{2}\right)\right]$. We will calculate the pumped electric and spin current from the left lead at zero temperature and set $V_{0}=69.7$. We set $x_{1}=-a$ and $x_{2}$ $=a / 10$. A gate voltage $v_{g}$ is applied in the double barrier

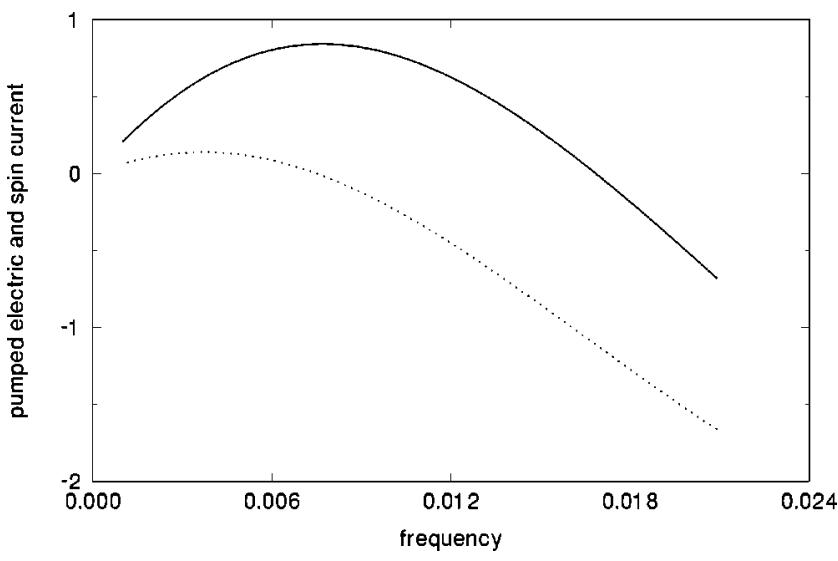

FIG. 1. The pumped electric current (solid line) and pumped spin current (dotted line) as a function of pumping frequency at $\theta=0$. Here $M=2$ and $E_{F}=3.4$. The vertical thin line corresponds to the frequency at which the pumped electric current is zero.

structure to control the resonant electron level. We assume that the Fermi level of the leads is in line with the resonant level at $v_{g}=0$. Finally the unit is set by $\hbar=2 m=q=2 a$ $=1$. For the system of $\mathrm{Fe} / \mathrm{Ge} / \mathrm{Fe}$ with $a=1000 \AA$, the energy unit is $E=0.046 \mathrm{meV}$, which corresponds to $\omega=11.0 \mathrm{GHz}$. The unit for pumped current is $2 \times 10^{-7} \mathrm{~A}$. The unit for spin current is $2 \mathrm{meV}$.

In Fig. 1, we show the pumped electric current (solid line) and spin current (dotted line) as functions of the pumping frequency when $\theta=0$ for a fixed Fermi energy and magnetization $M$. As the frequency increases, the pumped electric current increases and reaches a maximum value at $\omega=0.0077$. As the frequency increases further, the pumped electric current decreases and becomes negative at large frequency. In the presence of magnetic electrodes, the pumped electric current is spin polarized with a nonzero spin current. The behavior of the pumped spin current is similar to that of the pumped electric current. It is positive at a small pumping frequency and becomes negative at larger frequencies. At $\omega=0.0168$ (thin vertical line in Fig. 1), the pumped electric current is zero and a pure spin current is achieved. The physics behind this is the following. During parametric pumping, the system pumps out spin-up and -down electrons. For electron with a given Fermi energy, the potential of the ferromagnetic lead for spin-up electron is $-M$, while for spindown electron it is $M$. As a result, the pumped electric current for different spins can have different sign, i.e., a spin-up electron pumps out from the right lead to the left whereas a spin-down electron flows to the right lead during the pumping. This way a spin current is generated. At a certain value of the frequency, a complete cancellation of the electric current occurs and a pure spin current is delivered.

In Fig. 2 we plot the pumped electric current and spin current versus the pumping frequency for $\theta=\pi \cdot{ }^{24} \mathrm{We}$ observe that the pumped electric current for $\theta=0$ has the same order of magnitude as that at $\theta=\pi$. The pumped spin current, however, is quite different from that at $\theta=0$. It displays a linear dependency on the pumping frequency. The frequency at which a pure spin current occurs is also different from the case when $\theta=0$. Comparing with the case of $\theta=0$, the spin 


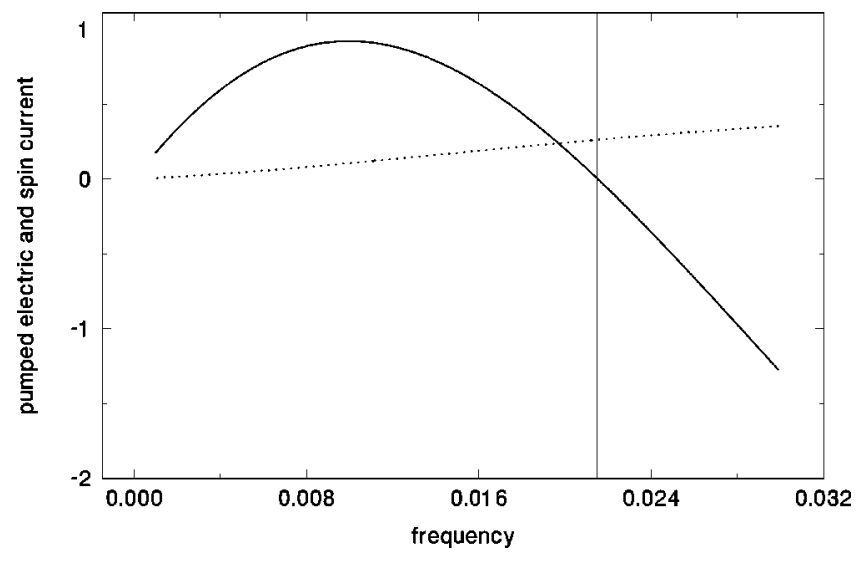

FIG. 2. The pumped electric current (solid line) and pumped spin current (dotted line) as a function of pumping frequency at $\theta=\pi$. Other parameters are the same as in Fig. 1 .

current reverses its direction when $\theta=\pi$. Our study shows that the pure spin current is a generic property and can occur at a wide range of parameters. In Fig. 1, we see that at $M$ $=2$ and $\omega=0.0168$ the system pumps out a pure spin current. As we vary $M$, the frequency $\omega$ at which the pure spin current occurs also changes. The trajectory or the "phase diagram" is depicted in Fig. 3, where the pumping frequency versus magnetization of the leads for $\theta=0$ is shown (solid line) along with the magnitude of pumped spin current.

In summary, we have proposed a nonadiabatic quantum spin pump which can generate a pure spin current with zero electric current during the parametric pumping. The device consists of a nonmagnetic system connected by two ferromagnetic leads. Since electrons with different spins experi-

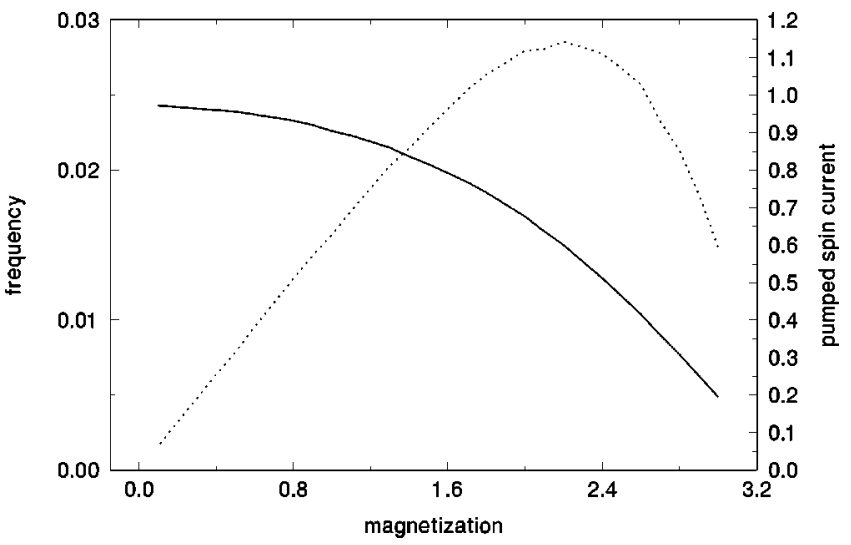

FIG. 3. The pumping frequency (solid line) and pumped spin current (dotted line) vs the magnetization of the ferromagnetic lead at which the pumped electric current is zero. For instance, when $B=1.6$ and $\omega=0.02$, the system pumps out the spin current $I_{s}$ $=0.96$.

ence different potential landscapes, the corresponding pumped current can be quite different. At certain conditions, the pumped electric current can be zero while a pure spin current is produced. Our numerical results show that this can be easily achieved by varying system parameters such as the pumping frequency. In this paper, we have not considered the spin orbit coupling ${ }^{25}$ which may affect our result. It may also lead to a new mechanism for spin pumping. ${ }^{26}$

We gratefully acknowledge support by a RGC grant from the SAR Government of Hong Kong under Grant No. HKU 7113/02P, a CRCG grant from the University of Hong Kong, and from NSERC of Canada and FCAR of Quebec (H.G).
*Electronic mail: jianwang@hkusub.hku.hk

${ }^{1}$ M. Switkes et al., Science 283, 1905 (1999).

${ }^{2}$ P.W. Brouwer, Phys. Rev. B 58, R10 135 (1998).

${ }^{3}$ F. Zhou et al., Phys. Rev. Lett. 82, 608 (1999).

${ }^{4}$ Y.D. Wei et al., Phys. Rev. B 62, 9947 (2000).

${ }^{5}$ T.A. Shutenko et al., Phys. Rev. B 61, 10366 (2000).

${ }^{6}$ J.E. Avron et al., Phys. Rev. Lett. 87, 236601 (2001).

${ }^{7}$ Y. Levinson et al., Physica A 302, 335 (2001).

${ }^{8}$ M. Moskalets and M. Buttiker, Phys. Rev. B 64, 201305 (2001); 66, 035306 (2002).

${ }^{9}$ M. Moskalets and M. Buttiker, Phys. Rev. B 66, 205320 (2002).

${ }^{10}$ Y.D. Wei et al., Phys. Rev. B 64, 115321 (2001); Y.D. Wei and J. Wang, ibid. 66, 195419 (2002).

${ }^{11}$ B.G. Wang et al., Phys. Rev. B 65, 073306 (2002).

${ }^{12}$ O. Entin-Wohlman and A. Aharony, Phys. Rev. B 66, 035329 (2002).

${ }^{13}$ M. Julliere, Phys. Lett. A 54, 225 (1975); R. Meservey and P.M. Tedrow, Phys. Rep. 238, 173 (1994); J.S. Moodera et al., Phys. Rev. Lett. 74, 3273 (1995); G.A. Prinz, Science 282, 1660
(1998); H. Mehrez et al., Phys. Rev. Lett. 84, 2682 (2000); A. Wolf et al., Science 294, 1488 (2001); N. Sergueev et al., Phys. Rev. B 65, 165303 (2002).

${ }^{14}$ A. Brataas et al., Phys. Rev. B 66, 060404 (2002).

${ }^{15}$ B.G. Wang et al., Phys. Rev. B 67, 092408 (2003).

${ }^{16}$ Q.F. Sun et al., Phys. Rev. Lett. 90, 258301 (2003); W. Lang, Q.F. Sun, H. Guo, and J. Wang, Appl. Phys. Lett. 83, 1397 (2003).

${ }^{17}$ E.R. Mucciolo et al., Phys. Rev. Lett. 89, 146802 (2002).

${ }^{18}$ S.K. Watson et al. cond-mat/0302492 (unpublished).

${ }^{19}$ J.C. Slonczewski, Phys. Rev. B 39, 6995 (1989).

${ }^{20}$ J.L. Wu et al., Phys. Rev. B 66, 205327 (2002).

${ }^{21}$ B.G. Wang et al., J. Phys. Soc. Jpn. 70, 2645 (2001).

${ }^{22}$ A.P. Jauho et al., Phys. Rev. B 50, 5528 (1994).

${ }^{23}$ M.K. Yip et al., Z. Phys. B: Condens. Matter 104, 463 (1997).

${ }^{24}$ After the gauge transformation, the spin current at $\theta=\pi$ changes the sign.

${ }^{25}$ S. Datta and B. Das, Appl. Phys. Lett. 56, 665 (1990).

${ }^{26}$ J. Schliemann, J.C. Egues, and D. Loss, Phys. Rev. Lett. 90, 146801 (2003). 